\title{
Comparison of the Calabi and Mabuchi geometries and applications to geometric flows
}

\author{
Tamás Darvas \\ University of Maryland, United States \\ Received 5 January 2016; received in revised form 9 September 2016; accepted 9 September 2016 \\ Available online 14 September 2016
}

\begin{abstract}
Suppose $(X, \omega)$ is a compact Kähler manifold. We introduce and explore the metric geometry of the $L^{p, q}$-Calabi Finsler structure on the space of Kähler metrics $\mathcal{H}$. After noticing that the $L^{p, q}$-Calabi and $L^{p^{\prime}}$-Mabuchi path length topologies on $\mathcal{H}$ do not typically dominate each other, we focus on the finite entropy space $\mathcal{E}^{\text {Ent }}$, contained in the intersection of the $L^{p}-C$ alabi and $L^{1}$-Mabuchi completions of $\mathcal{H}$ and find that after a natural strengthening, the $L^{p}$-Calabi and $L^{1}$-Mabuchi topologies coincide on $\mathcal{E}^{\text {Ent }}$. As applications to our results, we give new convergence results for the Kähler-Ricci flow and the weak Calabi flow.

(C) 2016 L'Association Publications de l'Institut Henri Poincaré. Published by Elsevier B.V. All rights reserved.
\end{abstract}

Keywords: Ricci flow; Kahler geometry; Calabi metric; Mabuchi metric

\section{Introduction and main results}

Suppose $\left(X^{n}, \omega\right)$ is a connected compact Kähler manifold. By $\mathcal{H}$ we denote the space of Kähler metrics $\omega^{\prime}$ that are cohomologous to $\omega$. In the 1950s Calabi initiated the study of the infinite-dimensional space $\mathcal{H}$, with the hopes of finding Kähler metrics with special curvature properties [9]. He introduced a Riemannian structure on $\mathcal{H}$ and formulated many related questions, including his famous conjecture ultimately solved by Yau [35]. Addressing one of Calabi's predictions, the path length completion of Calabi's Riemannian space was computed by Clarke-Rubinstein [14], and they also found a novel relation between Calabi geometry and convergence of the Kähler-Ricci (KR) flow on Fano manifolds. One of our purposes in the present paper is to further develop this circle of ideas. The KR flow was introduced by Hamilton [24], it satisfies the equation $\frac{d}{d t} \omega_{r_{t}}=\omega_{r_{t}}-\operatorname{Ric} \omega_{r_{t}}$ with $\omega \in c_{1}(X)$, and we refer to [6] for background, context and historic references.

In our first theorem, refining the findings of Clarke-Rubinstein $(p=2, q=1)$ [14] and also that of PhongSong-Sturm-Weinkove $(p=\infty, q=1)$ [28], we obtain the following convergence theorem for the KR flow, whose statement on the surface bears no connection with infinite-dimensional geometry:

E-mail address: tdarvas@math.umd.edu. 
Theorem 1.1. Suppose $1 \leq q \leq p \leq \infty, q \neq \infty$ and $\left(X^{n}, \omega\right)$ is Fano with $\omega \in c_{1}(X)$. Let $[0, \infty) \ni t \rightarrow \omega_{r_{t}} \in \mathcal{H}$ be a KR trajectory. Then a Kähler-Einstein metric in $\mathcal{H}$ exists if and only if

$$
\int_{0}^{\infty}\left\|n-S_{\omega_{r_{t}}}\right\|_{L^{p}\left(X,\left(\omega_{r_{t}}^{n} / \omega^{n}\right)^{q} \omega^{n}\right)} d t<\infty
$$

where $S_{\omega_{r_{t}}}$ is the scalar curvature of $\omega_{r_{t}}$. Additionally, if the above hold then $t \rightarrow r_{t}$ converges exponentially fast to a Kähler-Einstein metric.

As we shall see, the proof of this result requires no new a priori estimates, but instead rests on the observation that condition (1) is equivalent to saying that the KR trajectory $t \rightarrow \omega_{r_{t}}$ has finite length with respect to the $L^{p, q}-$ Calabi Finsler metric on $\mathcal{H}$, that we introduce now. By Hodge theory, if $\omega^{\prime} \in \mathcal{H}$ then $\omega^{\prime}=\omega_{u}:=\omega+i \partial \bar{\partial} u$ for some $u \in$ $C^{\infty}(X)$, hence $\mathcal{H}$ can be identified with $\mathcal{H}_{\omega}$, the set of normalized Kähler potentials:

$$
\mathcal{H}_{\omega}:=\left\{u \in \mathcal{C}^{\infty}(X): \omega_{u}=\omega+i \partial \bar{\partial} u>0, \int_{X} u \omega^{n}=0\right\} \simeq \mathcal{H} .
$$

As our approach in this note makes heavy use of pluripotential theory, we will mostly work with potentials instead of metrics. Treating $\mathcal{H}_{\omega}$ as a (trivial) Frèchet manifold, one can introduce the $L^{p, q}$-Calabi Finsler metric for arbitrary $1 \leq q \leq p<\infty$ :

$$
\|\beta\|_{p, q, u}^{C}=\left[\frac{1}{V} \int_{X}\left|\Delta_{\omega_{u}} \beta\right|^{p}\left[\frac{\omega_{u}{ }^{n}}{\omega^{n}}\right]^{q} \omega^{n}\right]^{1 / p}, \beta \in T_{u} \mathcal{H}_{\omega}, u \in \mathcal{H}_{\omega},
$$

where $V=\int_{X} \omega^{n}$ is the total volume. The case $p=2, q=1$ gives the Riemannian structure of Calabi [9] recently studied extensively in $[12,14]$. In the most important case $q=1$, we will simply refer to the metric in (2) as the $L^{p}$-Calabi metric.

Using this Finsler metric it is possible to compute the length of smooth curves, and introduce the associated path length pseudometric $d_{p, q}^{C}$ on $\mathcal{H}_{\omega}$. It turns out that $\left(\mathcal{H}_{\omega}, d_{p, q}^{C}\right)$ is a bona fide metric space and its completion and metric growth can be analytically characterized using elements from the finite energy pluripotential theory of GuedjZeriahi [23], generalizing [14, Theorem 5.4] in the process:

Theorem 1.2. Suppose $\left(X^{n}, \omega\right)$ is Kähler and $1 \leq q \leq p<\infty$. Then $\left(\mathcal{H}_{\omega}, d_{p, q}^{C}\right)$ is a metric space whose completion is $\left(\mathcal{E}^{L^{q}}, d_{p, q}^{C}\right)$. Characterizing convergence, a sequence $\left\{u_{j}\right\}_{j} \subset \mathcal{H}_{\omega}$ is $d_{p, q}^{C}$-Cauchy if and only if

$$
\int_{X}\left|\frac{\omega_{u_{j}}^{n}}{\omega^{n}}-\frac{\omega_{u_{k}}^{n}}{\omega^{n}}\right|^{q} \omega^{n} \rightarrow 0 \text { as } j, k \rightarrow \infty .
$$

Additionally, $\left(\mathcal{E}^{L^{1}}, d_{2,1}^{C}\right)$ is a $\mathrm{CAT}(1 / 4)$ geodesic metric space.

This theorem seems to give the first geometric characterization of the well known potential space $\mathcal{E}^{L^{q}}$. Roughly speaking, $\mathcal{E}^{L^{q}}$ contains degenerate metrics with volume measure having $L^{q}$-density, and we give now the precise definition, referring to [23] for additional details. In [23], associated to any $u \in \operatorname{PSH}(X, \omega)$ the authors introduce a non-pluripolar measure $\omega_{u}^{n}$ on $X$, satisfying $\int_{X} \omega_{u}^{n} \leq \int_{X} \omega^{n}$, generalizing the usual complex Monge-Ampère measure of Bedford-Taylor in case $u$ is additionally bounded. By definition, $u \in \mathcal{E} \subset \operatorname{PSH}(X, \omega)$ if $u$ has "full volume", i.e., $\int_{X} \omega_{u}^{n}=\int_{X} \omega^{n}$. Given $p, q \geq 1$, two important subclasses of potentials inside $\mathcal{E}$ are as follows:

$$
\mathcal{E}^{L^{q}}:=\left\{u \in \mathcal{E}, \int_{X} u \omega^{n}=0, \frac{\omega_{u}^{n}}{\omega^{n}} \in L^{q}\left(X, \omega^{n}\right)\right\}, \quad \mathcal{E}^{p}:=\left\{u \in \mathcal{E}, \int_{X} u \omega^{n}=0, \int|u|^{p} \omega_{u}^{n}<\infty\right\} .
$$

Remark 1.3. By basic analysis, the $d_{p, q}^{C}$-convergence characterization (3) extends to sequences inside the completion $\mathcal{E}^{L^{q}}$ as well. 
One can derive the equation for $L^{p, q}$-Calabi geodesics by computing the variation of the Finsler energy along curves with fixed endpoints. Contrary to the findings of [12] in the particular case $p=2, q=1$, this geodesic equation does not admit smooth solutions for general $p$ and $q$, and to avoid complications we omit the discussion of "weak geodesics" in this note.

A distinguishing feature of the $L^{p, q}$-Calabi geometry is that the associated path length metric $d_{p, q}^{C}$ induces the same completion on $\mathcal{H}_{\omega}$ for all $p \geq 1$ and fixed $q$, even though the corresponding Finsler metrics for different $p$ are not even fiberwise conformally equivalent. Indeed, as follows from (3), $d_{p, q}^{C}$-convergence does not depend on the value of $p$. We are not aware of other families of infinite dimensional Finsler structures that would enjoy this same property. See [15] for a treatment of conformal deformations of the Ebin metric on the space of Riemannian metrics, where a related but different phenomenon occurs.

As we learned after the completion of this paper, in [13, Section 4], motivated by different goals, a family of Riemannian metrics was introduced and studied in detail that overlaps with our construction of $L^{p, q}$-Calabi metrics when $p=2$.

On top of applications to geometric flows, our motivation for studying the $L^{p, q}$ generalization of Calabi's Riemannian structure comes from the corresponding $L^{p}$ generalization of the Mabuchi geometry on $\mathcal{H}$ [18], that led to many applications in the study of canonical Kähler metrics $[1,2,19,20]$, and Theorem 1.2 stands in direct analogy with the findings of $[17,18]$ that we recall now. The $L^{p}$-Mabuchi Finsler metric of $\mathcal{H}_{\omega}$ is defined as follows:

$$
\|\phi\|_{p, u}=\left[\frac{1}{V} \int_{X}\left|\phi-\bar{\phi}_{\omega_{u}}\right|^{p} \omega_{u}^{n}\right]^{1 / p}, \phi \in T_{u} \mathcal{H}_{\omega}, u \in \mathcal{H}_{\omega},
$$

where $\bar{\phi}_{\omega_{u}}=\frac{1}{V} \int_{X} \phi \omega_{u}^{n}$. The case $p=2$ gives the Riemannian structure of Mabuchi-Semmes-Donaldson [26,31,22], a space with non-positive sectional curvature, with close ties to canonical Kähler metrics. As shown in [19,20], the case $p=1$ gives a geometry with good compactness properties, suitable for the variational study of canonical metrics by way of infinite-dimensional convex optimization.

As in the case of the $L^{p, q}$-Calabi metric, using the Finsler structure of (4) we can measure the length of smooth curves, and for the associated path length pseudometric $d_{p}^{M}$ on $\mathcal{H}_{\omega}$ we recall now the Mabuchi analog of Theorem 1.2, concatenating the relevant parts of [17, Theorem 1] and [18, Theorem 2, Theorem 3]. We refer to [17,18] for further details.

Theorem 1.4. Suppose $\left(X^{n}, \omega\right)$ is Kähler and $p \geq 1$. Then $\left(\mathcal{H}_{\omega}, d_{p}^{M}\right)$ is a metric space whose completion is $\left(\mathcal{E}^{p}, d_{p}^{M}\right)$. Characterizing convergence, a sequence $\left\{u_{j}\right\}_{j} \subset \mathcal{H}_{\omega}$ is $d_{p}^{M}$-Cauchy if and only if

$$
\int_{X}\left|u_{j}-u_{k}\right|^{p} \omega_{u_{j}}^{n}+\int_{X}\left|u_{j}-u_{k}\right|^{p} \omega_{u_{k}}^{n} \rightarrow 0 \text { as } j, k \rightarrow \infty .
$$

Additionally, $\left(\mathcal{E}_{M}^{2}, d_{2}^{M}\right)$ is a $\mathrm{CAT}(0)$ geodesic metric space.

With Theorems 1.2 and 1.4 in hand, we can answer in a much more general context the questions of ClarkeRubinstein [14, Section 7.2], who proposed to compare the $L^{2}$-Mabuchi and $L^{2}$-Calabi metric structures. It follows from the proof of Theorem 1.2 that $\left(\mathcal{H}_{\omega}, d_{p, 1}^{C}\right)$ has finite diameter. As $\left(\mathcal{H}_{\omega}, d_{p^{\prime}}^{M}\right)$ has infinite diameter [18], it is not possible for $d_{p, 1}^{C}$ to globally dominate $d_{p^{\prime}}^{M}$. Even in the absence of global metric domination, one may still hope that domination holds on the level of the induced topologies. In case $q>1$, thanks to strong estimates of Kolodziej [25, p. 668], the $d_{p, q}^{C}$-topology dominates the $C^{0}$-topology hence implicitly also the $d_{p^{\prime}}^{M}$-topology. However the case $q=1$ is much more delicate and does not allow any kind of domination (see [3, Theorem 1.3] for related a priori estimates), as we summarize in the following result that fully characterizes the relationship between the $L^{p, q}$-Calabi and $L^{p^{\prime}}$-Mabuchi topologies:

Theorem 1.5. Suppose $(\mathcal{H}, \omega)$ is Kähler, $1 \leq q \leq p<\infty$ and $1 \leq p^{\prime}$. The following hold:

(i) There exists a sequence $\left\{v_{k}\right\}_{k \in \mathbb{N}} \subset \mathcal{H}_{\omega}$ that is $d_{p^{\prime}}^{M}$-Cauchy but does not contain any $d_{p, q}^{C}$-Cauchy subsequences. 
(ii) If $q>1$, then any $d_{p, q}^{C}$-Cauchy sequence inside $\mathcal{H}_{\omega}$ is $d_{p^{\prime}}^{M}$-Cauchy as well.

(iii) There exists a sequence $\left\{u_{k}\right\}_{k \in \mathbb{N}} \subset \mathcal{H}_{\omega}$ that is $d_{p, 1}^{C}$-Cauchy but does not contain any $d_{p^{\prime}}^{M}$-Cauchy subsequences.

For the rest of the introduction, let us focus on the case $q=1$, most important from the point of view of geometric applications. An important step in the proof of the previous theorem is noticing that the completion of $\mathcal{H}_{\omega}$ with respect to the $L^{p}$-Calabi and $L^{p^{\prime}}$-Mabuchi metrics cannot contain each other. Though containment is not possible, it is natural to search for interesting subspaces of the intersection $\overline{\left(\mathcal{H}_{\omega}, d_{p^{\prime}}^{M}\right)} \cap \overline{\left(\mathcal{H}_{\omega}, d_{p, 1}^{C}\right)}=\mathcal{E}^{p^{\prime}} \cap \mathcal{E}^{L^{1}}$.

Given the importance of the $L^{1}$-Mabuchi metric in applications to existence/uniqueness of canonical Kähler metrics $[20,19,1]$, we further restrict attention to the case $p \geq 1$ and $p^{\prime}=1$. A natural subspace of the intersection $\mathcal{E}^{1} \cap \mathcal{E}^{L^{1}}$ is the space of potentials with finite entropy, studied in $[7,2]$ in connection with canonical Kähler metrics:

$$
\mathcal{E}^{\text {Ent }}:=\left\{u \in \mathcal{E}: \operatorname{Ent}\left(\omega^{n}, \omega_{u}^{n}\right)<\infty, \int_{X} u \omega^{n}=0\right\},
$$

where $\operatorname{Ent}\left(\omega^{n}, \omega_{u}^{n}\right)=\infty$ if $\omega_{u}^{n}$ is not absolutely continuous with respect to $\omega^{n}$, and is equal to $\int_{X} \log \left(\frac{\omega_{u}^{n}}{\omega^{n}}\right) \frac{\omega_{u}^{n}}{\omega^{n}} \omega^{n}$ otherwise. By definition, $\mathcal{E}^{\text {Ent }} \subset \mathcal{E}^{L^{1}}$ and it is well known that also $\mathcal{E}^{\text {Ent }} \subset \mathcal{E}^{1}$ (see [7,2]), hence as proposed

$$
\mathcal{E}^{\text {Ent }} \subset \mathcal{E}^{1} \cap \mathcal{E}^{L^{1}} \text {. }
$$

It follows that $\mathcal{E}^{\text {Ent }}$ can be endowed with two different non-complete topologies induced by $d_{p, 1}^{C}$ and $d_{1}^{M}$. A natural way to make these topologies complete on $\mathcal{E}^{\text {Ent }}$ is to strengthen them enough to make the map $u \rightarrow \operatorname{Ent}\left(\omega^{n}, \omega_{u}^{n}\right)$ continuous. It turns out this procedure gives equivalent topologies and in fact much more is true:

Theorem 1.6. Suppose $u_{j}, u \in \mathcal{E}^{\mathrm{Ent}}$ satisfy $\operatorname{Ent}\left(\omega^{n}, \omega_{u_{j}}^{n}\right) \rightarrow \operatorname{Ent}\left(\omega^{n}, \omega_{u}^{n}\right)$. Then the following are equivalent:

(i) $u_{j} \rightarrow u$ in $L^{1}\left(X, \omega^{n}\right)$.

(ii) $\omega_{u_{j}}^{n} \rightarrow \omega_{u}^{n}$ in the weak sense of measures.

(iii) $d_{1}^{M}\left(u_{j}, u\right) \rightarrow 0$.

(iv) $d_{p, 1}^{C}\left(u_{j}, u\right) \rightarrow 0$.

An immediate application of the equivalence between (iii) and (iv) in the last theorem and [2, Theorem 1.2, Theorem 1.11] is a new convergence result for the weak Calabi flow. This weak flow is a generalization of the classical smooth Calabi flow $[10,11]$ (that is governed by the equation $\frac{d}{d t} c_{t}=S_{\omega_{c_{t}}}-\bar{S}$ ) and was initially introduced and studied by Streets in the context of the abstract metric completion $\overline{\left(\mathcal{H}, d_{2}^{M}\right)}$ [32,33]. A better understanding of this latter space in [17] led to more precise long time convergence and asymptotics results for the weak Calabi flow in [2] and for more details, related terminology and historic references we refer to this last paper. We note the following corollary, which is a direct consequence of Theorem 1.6 above and [2, Theorem 1.5, Theorem 1.11]:

Corollary 1.7. Suppose $p \geq 1$ and there exists a constant scalar curvature metric in $\mathcal{H}$. Then, given any weak Calabi flow trajectory $[0, \infty) \ni t \rightarrow c_{t} \in \mathcal{E}^{2}$, there exists a constant scalar curvature potential $c_{\infty} \in \mathcal{H}_{\omega}$ such that $d_{p, 1}^{C}\left(c_{t}, c_{\infty}\right) \rightarrow 0$, in particular $\int_{X}\left|\frac{\omega_{t}^{n}}{\omega^{n}}-\frac{\omega_{c \infty}^{n}}{\omega^{n}}\right| \omega^{n} \rightarrow 0$.

\section{The $L^{p, q}$-Calabi geometry of $\mathcal{H}_{\omega}$}

As we will see, $\mathcal{H}_{\omega}$ equipped with the $L^{p, q}$-Calabi Finsler structure can be embedded isometrically into $L^{p}\left(X, \omega^{n}\right)$. For this reason, we focus on the "flat" geometry of $L^{p}\left(X, \omega^{n}\right)$ in the next short subsection.

\section{1. $L^{p}$-geometry on $L^{p / q}$-spheres}

Let $1 \leq q \leq p<\infty$ and $X$ be a compact manifold with a positive Borel measure $\mu$ satisfying $\mu(X)=\int_{X} \mu<\infty$. As a Fréchet manifold, $C^{\infty}(X)$ can be equipped with the trivial $L^{p}$-Finsler structure: 


$$
\|\psi\|_{p, f}=\left(\frac{1}{\mu(X)} \int_{X}|\psi|^{p} \mu\right)^{1 / p}, \psi \in T_{f} C^{\infty}(X) \simeq C^{\infty}(X), f \in C^{\infty}(X) .
$$

It is a classical fact that straight segments joining various points of $C^{\infty}(X)$ are geodesics for this metric. The $L^{p / q}$-sphere with radius $r$ is denoted by:

$$
\mathbb{S}_{L^{p / q}}(\mu, r)=\left\{f \in C^{\infty}(X) \text { s.t. } \frac{1}{\mu(X)} \int_{X}|f|^{p / q} \mu=r^{p / q}\right\} .
$$

Unfortunately, for most $p$ and $q$, the $L^{p / q}$-sphere is not even a smooth submanifold of $C^{\infty}(X)$, however if we restrict to the "octant" $\mathbb{S}_{L^{p / q}}^{+}(\mu, r)=\mathbb{S}_{L^{p / q}}(\mu, r) \cap\{f>0\}$, we do get a smooth submanifold. As such, one can pullback the $L^{p}$-Finsler metric of $(6)$ to $\mathbb{S}_{L^{p / q}}^{+}(\mu, r)$, and study the resulting path length metric space $\left(\mathbb{S}_{L^{p / q}}^{+}(\mu, r), d_{p, q}^{\mathbb{S}^{+}}\right)$. We will need the following basic result in this direction, which roughly says that the "chordal metric" is equivalent to the "round metric" on $\mathbb{S}_{L p / q}^{+}(\mu, r)$ :

Proposition 2.1. Fix $f \in \mathbb{S}_{L^{p / q}}^{+}(\mu, r)$. Then there exists $C:=C(\mu, p, q, f, r)>0$ such that for any $f_{0}, f_{1} \in$ $\mathbb{S}_{L^{p / q}}^{+}(\mu, r)$ the following holds:

$$
\frac{C}{d_{p, q}^{\mathbb{S}^{+}}\left(f, f_{0}\right)+d_{p, q}^{\mathbb{S}^{+}}\left(f, f_{1}\right)+1} d_{p, q}^{\mathbb{S}^{+}}\left(f_{0}, f_{1}\right) \leq\left(\frac{1}{\mu(X)} \int_{X}\left|f_{0}-f_{1}\right|^{p} \mu\right)^{1 / p} \leq d_{p, q}^{\mathbb{S}^{+}}\left(f_{0}, f_{1}\right) .
$$

Proof. The inequality $\left(\frac{1}{\mu(X)} \int_{X}\left|f_{0}-f_{1}\right|^{p} \mu\right)^{1 / p} \leq d_{p, q}^{\mathbb{S}^{+}}\left(f_{0}, f_{1}\right)$ follows from the fact that $[0,1] \ni t \rightarrow f_{t}:=$ $f_{0}+t\left(f_{1}-f_{0}\right) \in C^{\infty}(X)$ is a geodesic in $C^{\infty}(X)$ with respect to the $L^{p}$-Finsler metric and has length equal to $\left(\frac{1}{\mu(X)} \int_{X}\left|f_{0}-f_{1}\right|^{p} \mu\right)^{1 / p}$. Any curve joining $f_{0}, f_{1}$ inside $\mathbb{S}_{L^{p / q}}^{+}(\mu, r)$ has to have length less than $t \rightarrow f_{t}$.

For the other inequality, we will estimate the length of the curve

$$
[0,1] \ni t \rightarrow \alpha_{t}:=\frac{r f_{t}}{\left\|f_{t}\right\|_{p / q}}=\frac{r\left(f_{0}+t\left(f_{1}-f_{0}\right)\right)}{\left\|f_{0}+t\left(f_{1}-f_{0}\right)\right\|_{p / q}} \in \mathbb{S}_{L^{p / q}}^{+}(\mu, r)
$$

joining $f_{0}, f_{1}$. Note that the denominator of the expression above is nonzero, as $f_{0}, f_{1}>0$. Using that

$$
\dot{\alpha}_{t}=\frac{r\left(f_{1}-f_{0}\right)}{\left\|f_{t}\right\|_{p / q}}-\frac{r f_{t}}{\left\|f_{t}\right\|_{p / q}^{p / q+1}} \int_{X}\left(f_{1}-f_{0}\right) f_{t}^{p / q-1} d \mu,
$$

we have the following sequence of estimates:

$$
\begin{aligned}
\int_{0}^{1}\left\|\dot{\alpha}_{t}\right\|_{p} d t & \leq \int_{0}^{1} \frac{r\left\|f_{1}-f_{0}\right\|_{p}}{\left\|f_{t}\right\|_{p / q}} d t+\int_{0}^{1} \frac{r\left\|f_{t}\right\|_{p}}{\left\|f_{t}\right\|_{p / q}^{p / q+1}} \int_{X}\left|f_{1}-f_{0}\right| f_{t}^{p / q-1} d \mu d t \\
& \leq \int_{0}^{1} \frac{r\left\|f_{1}-f_{0}\right\|_{p}}{\left\|f_{t}\right\|_{p / q}} d t+\int_{0}^{1} \frac{r\left\|f_{t}\right\|_{p}}{\left\|f_{t}\right\|_{p / q}^{p / q+1}}\left\|f_{1}-f_{0}\right\|_{p / q}\left\|f_{t}\right\|_{p / q}^{p / q-1} d t \\
& \leq r\left\|f_{1}-f_{0}\right\|_{p} \int_{0}^{1} \frac{1}{\left\|f_{t}\right\|_{p / q}} d t+C^{\prime}(\mu, p, q)\left\|f_{1}-f_{0}\right\|_{p} \int_{0}^{1} \frac{\left\|f_{t}\right\|_{p}}{\left\|f_{t}\right\|_{p / q}^{2}} d t \\
& \leq r\left\|f_{1}-f_{0}\right\|_{p} \int_{0}^{1} \frac{1}{\left\|f_{t}\right\|_{p / q}}+C^{\prime}(\mu, p, q) \frac{(1-t)\left\|f-f_{0}\right\|_{p}+t\left\|_{f}-f_{1}\right\|_{p}+\|f\|_{p}}{\left\|f_{t}\right\|_{p / q}^{2}} d t \\
& \leq C(\mu, p, q, r, f)\left(d_{p, q}^{\mathbb{S}^{+}}\left(f, f_{0}\right)+d_{p, q}^{\mathbb{S}^{+}}\left(f, f_{1}\right)+1\right)\left\|f_{1}-f_{0}\right\|_{p},
\end{aligned}
$$

where to obtain the second line we have used the Hölder inequality with exponents $p / q \geq 1$ and $(p / q) /(p / q-1) \geq 1$ in the last integrand. To get the third line, we have used that $\left\|f_{0}-f_{1}\right\|_{p / q} \leq C^{\prime}(\mu, p, q)\left\|f_{0}-f_{1}\right\|_{p}$. For the fourth line, 
we have used the triangle inequality for the $L^{p}$-norm. To get the last line, we have used that $\left\|f-f_{0}\right\|_{p} \leq d_{p, q}^{\mathbb{S}^{+}}\left(f, f_{0}\right)$, $\left\|f-f_{1}\right\|_{p} \leq d_{p, q}^{\mathbb{S}^{+}}\left(f, f_{1}\right), f_{t} \geq f_{0} / 2 \geq 0$ for $t \in[0,1 / 2]$ and $f_{t} \geq f_{1} / 2 \geq 0$ for $t \in[1 / 2,1]$, hence $\left\|f_{t}\right\|_{p / q} \geq r / 2$ for all $t \in[0,1]$. To finish the proof, we conclude:

$$
d_{p, q}^{\mathbb{S}^{+}}\left(f_{0}, f_{1}\right) \leq \int_{0}^{1}\left\|\dot{\alpha}_{t}\right\|_{p} d t \leq C(\mu, p, q, f)\left(d_{p, q}^{\mathbb{S}^{+}}\left(f, f_{0}\right)+d_{p, q}^{\mathbb{S}^{+}}\left(f, f_{1}\right)+1\right)\left\|f_{1}-f_{0}\right\|_{p}
$$

\subsection{Proof of Theorem 1.2 and Theorem 1.1}

Proof of Theorem 1.2. To start the proof, we notice that for arbitrary $1 \leq q \leq p<\infty$ the infinite-dimensional Finsler manifolds $\left(\mathcal{H}_{\omega},\|\cdot\|_{p, q,(\cdot)}^{C}\right)$ and $\left(\mathbb{S}_{L^{p / q}}^{+}\left(\omega^{n}, p / q\right),\|\cdot\|_{p,(\cdot)}\right)$ are isometric via the map $F: \mathcal{H}_{\omega} \rightarrow \mathbb{S}_{L^{p / q}}^{+}\left(\omega^{n}, p / q\right)$, given by the formula

$$
F(u):=\frac{p}{q}\left(\frac{\omega_{u}^{n}}{\omega^{n}}\right)^{\frac{q}{p}} .
$$

By the Calabi-Yau theorem, the map $F$ is bijective. As $F(u)_{*}(\delta u)=\left(\omega_{u}^{n} / \omega^{n}\right)^{q / p} \Delta_{\omega_{u}} \delta u$, by inspection we see that $F^{*}\|\cdot\|_{p, F(\cdot)}=\|\cdot\|_{p, q,(\cdot)}^{C}$. All this implies that

$$
d_{p, q}^{C}\left(u_{0}, u_{1}\right)=d_{p, q}^{\mathbb{S}^{+}}\left(F\left(u_{0}\right), F\left(u_{1}\right)\right),
$$

in particular, (7) gives that $d_{p, q}^{C}$ is indeed a metric on $\mathcal{H}_{\omega}$. From (7) it also follows that $\left\{u_{j}\right\}_{j} \subset \mathcal{H}_{\omega}$ is $d_{p, q}^{C}$-Cauchy if and only if

$$
\int_{X}\left|\left(\frac{\omega_{u_{j}}^{n}}{\omega^{n}}\right)^{q / p}-\left(\frac{\omega_{u_{k}}^{n}}{\omega^{n}}\right)^{q / p}\right|^{p} \omega^{n} \rightarrow 0, j, k \rightarrow \infty .
$$

Using this, Lemma 2.2 below implies that $\left\{u_{j}\right\}_{j}$ is $d_{p, q}^{C}$-Cauchy if and only if (3) holds. The identification $\overline{\left(\mathcal{H}_{\omega}, d_{p, q}^{C}\right)}=\mathcal{E}^{L^{q}}$ readily follows as well.

Lastly, we focus on the case $p=2, q=1$ extensively treated in [12,14]. As observed in [12, Theorem 1.1] (see also the discussion following [14, Remark 4.2]), the Riemannian space $\left(\mathcal{H}_{\omega},\|\cdot\|_{2,1,(\cdot)}^{C}\right)$ has positive constant sectional curvature equal to $1 / 4$, what is more, any two points of $\mathcal{H}_{\omega}$ can be joined by a Riemannian geodesic. Roughly, this follows from the fact that $\left(\mathcal{H}_{\omega},\|\cdot\|_{2,1,(\cdot)}^{C}\right)$ is isometric to $\left(\mathbb{S}_{L^{2}}^{+}\left(\omega^{n}, 2\right),\|\cdot\|_{2,(\cdot)}\right)$, which is a totally geodesic open subset of an infinite-dimensional sphere with radius 2.

Given $u, v, w \in \mathcal{H}_{\omega}$, let $U=F(u), V=F(v), W=F(w) \in \mathbb{S}_{L^{2}}^{+}\left(\omega^{n}\right)$. Also let $\mathcal{V} \subset L^{2}\left(X, \omega^{n}\right)$ be the 3 dimensional subspace spanned by $U, V, W$ and $\mathbb{S}_{U V W}=\mathcal{V} \cap \mathbb{S}_{L^{2}}^{+}\left(\omega^{n}, 2\right)$. Together with the induced Riemannian metric, $\mathbb{S}_{U V W}$ is isometric to an open subset of the 2-dimensional round sphere with its round metric, hence it has constant sectional curvature $1 / 4$. The geodesic triangle $U V W$ of $\mathbb{S}_{L^{2}}^{+}\left(\omega^{n}, 2\right)$, with edges at $U, V, W$ lies inside $\mathbb{S}_{U V W}[12$, Theorem 1.4]. As $\mathbb{S}_{U V W}$ is a model space with constant scalar curvature equal to $1 / 4$, the geodesic triangle $U V W$ (lying inside it) has to satisfy the CAT(1/4) inequality [8], ultimately giving that $\left(\mathcal{H}_{\omega}, d_{2}^{C}\right)$ is a CAT(1/4) space.

To finish the proof, we can use $\left[8\right.$, Corollary 3.11, p. 187] to conclude that the metric completion $\overline{\left(\mathcal{H}_{\omega}, d_{2}^{C}\right)}=$ $\left(\mathcal{E}^{L^{1}}, d_{2}^{C}\right)$ is a CAT(1/4) geodesic metric space as well.

As promised in the above proof, let us state the following measure theoretic lemma, whose proof uses the classical Vitali convergence theorem [29, Theorem 8.5.14], and is exactly the same as the argument of [14, Lemma 5.3]:

Lemma 2.2. Suppose $f_{j}, f \in L^{q}\left(X, \omega^{n}\right)$ with $f_{j}, f \geq 0$. Then $\left\|f_{j}-f\right\|_{L^{q}} \rightarrow 0$ if and only if $\left\|f_{j}^{q / p}-f^{q / p}\right\|_{L^{p}} \rightarrow 0$.

Lastly, we provide the following theorem, which contains Theorem 1.1 as a particular case. We refer to [18,19,27] for analogous results on the $L^{p^{\prime}}$-Mabuchi convergence of the Kähler-Ricci flow. 
Theorem 2.3. Suppose $\left(X^{n}, \omega\right)$ is Fano with $[\omega]=-c_{1}\left(K_{X}\right)$ and $1 \leq q \leq p \leq \infty$. Suppose $[0, \infty) \ni t \rightarrow r_{t} \in \mathcal{H}_{\omega}$ is a Kähler-Ricci trajectory. Then the following are equivalent:

(i) There exists a Kähler-Einstein potential inside $\mathcal{H}_{\omega}$.

(ii) $t \rightarrow r_{t}$ converges $C^{\infty}$-exponentially fast to some Kähler-Einstein potential $r_{\infty} \in \mathcal{H}_{\omega}$.

(iii) $t \rightarrow r_{t}$ has finite $d_{p, 1}^{C}$-length, i.e., $\int_{0}^{\infty}\left\|n-S_{\omega_{r_{t}}}\right\|_{L^{p}\left(X,\left(\omega_{r_{t}}^{n} / \omega^{n}\right)^{q} \omega^{n}\right)}<\infty$.

(iv) $\left\{r_{t}\right\}_{t \geq 0} \subset \mathcal{H}_{\omega}$ forms a $d_{p, q}^{C}$-Cauchy sequence.

Proof. Let us first assume that $p \neq \infty$. If (i) holds then by results of Perelman, Tian-Zhu, Phong-Song-SturmWeinkove and Collins-Székelyhidi $[34,28,16]$ imply that the Kähler-Ricci trajectory $t \rightarrow r_{t}$ converges $C^{\infty}$ exponentially fast to some Kähler-Einstein potential $r_{\infty} \in \mathcal{H}_{\omega}$, hence (ii) holds. $C^{\infty}$-exponential convergence of $t \rightarrow r_{t}$ implies the finiteness of the integral in (iii). Condition (iii) implies (iv) trivially.

We are left to show that (iv) implies (i). The ideas of [14, Corollary 6.7] apply again, but we give here a slightly different argument. Suppose (iv) holds but (i) does not. Let $r_{\infty} \in \mathcal{E}^{L^{q}}$ be the $d_{p, q}^{C}$-limit of $r_{t}$. It follows from the convergence criterion of (3) and Remark 3.2 below that we also have weak convergence on the level of potentials, namely $r_{t} \rightarrow{ }_{L^{1}\left(X, \omega^{n}\right)} r_{\infty}$. On the other hand, by [30, Theorem 1.3], there exists $t_{j} \rightarrow \infty$ and $\psi \in \operatorname{PSH}(X, \omega)$ such that $\psi$ has proper multiplier ideal sheaf, in particular by Skoda's theorem $\psi$ has non-zero Lelong number at some $x \in X$. By [23, Corollary 1.8] this implies that $\psi \notin \mathcal{E}$. But by uniqueness of $L^{1}\left(X, \omega^{n}\right)$-limits, we have $\psi=r_{\infty} \in \mathcal{E}^{L^{q}} \subset \mathcal{E}$, a contradiction.

Now we deal with the case $p=\infty$. Clearly, the directions (i) $\rightarrow$ (ii) $\rightarrow$ (iii) $\rightarrow$ (iv) still hold. To prove that (iv) implies (i) we just need to notice that $d_{\infty}^{C}$-convergence trivially implies $d_{p, q}^{C}$-convergence for any $1 \leq q \leq p<$ $\infty$.

\section{3. $L^{p, q}$-Calabi vs. $L^{p^{\prime}}$-Mabuchi geometry}

\subsection{Proof of Theorem 1.5}

To prove (i), we recall first that $\mathcal{E}^{p^{\prime}} \not \subset \mathcal{E}^{L^{q}}$. Indeed, we can choose $v_{0}, v_{1} \in \mathcal{H}_{\omega}$ such that the level set $\left\{v_{0}-v_{1}=0\right\}$ does not contain critical points of $v_{0}-v_{1}$. Then a basic calculation yields that the bounded potential $u=\max \left(v_{0}, v_{1}\right)-$ $\int_{X} \max \left(v_{0}, v_{1}\right) \omega^{n}$ satisfies $u \in \mathcal{E}^{p^{\prime}} \backslash \mathcal{E}^{L^{q}}$, because $\omega_{u}^{n}$ charges the hypersurface $\left\{v_{0}=v_{1}\right\}$, a set of Lebesgue measure zero.

Now let $u \in \mathcal{E}^{p^{\prime}} \backslash \mathcal{E}^{L^{q}}$ arbitrary. By Theorem 1.4 there exists $u_{j} \in \mathcal{H}_{\omega}$ such that $d_{p^{\prime}}^{M}\left(u_{j}, u\right) \rightarrow 0$. This in particular gives that $\omega_{u_{j}}^{n} \rightarrow \omega_{u}^{n}$ weakly [18, Theorem 5(i)]. We claim that $\left\{u_{j}\right\}_{j}$ cannot contain a $d_{p, q}^{C}$-Cauchy subsequence. Indeed, if this were the case, then by Theorem 1.2 above, for some subsequence of $u_{j}$, again denoted by $u_{j}$, the densities $\omega_{u_{j}}^{n} / \omega^{n}$ would converge in $L^{q}\left(X, \omega^{n}\right)$ to some $f \in L^{q}(X, \omega)$. But as $\omega_{u_{j}}^{n} \rightarrow \omega_{u}^{n}$ weakly, this would imply that $\omega_{u}^{n} / \omega^{n}=f \in L^{q}\left(X, \omega^{n}\right)$, a contradiction with $u \in \mathcal{E}^{p^{\prime}} \backslash \mathcal{E}^{L^{q}}$.

To argue (iii), we first show that $\mathcal{E}^{L^{1}} \not \subset \mathcal{E}^{p^{\prime}}$. This is again likely known to experts, however we could not find an exact reference, so we give a construction allowing a great amount of flexibility. Let $u \in \mathcal{E}^{p^{\prime}}, u \leq-1$ and unbounded such that for each set $U_{k}=\left\{k<|u|^{p^{\prime}} \leq k+1\right\}$ we have $\omega^{n}\left(U_{k}\right)>0, k \geq 1$. By the construction in [23, Example 2.14] (see also [4, Proposition 5]), such $u$ can be found. We introduce $f \in L^{1}\left(X, \omega^{n}\right)$ :

$$
f(x)=\sum_{k \geq 1} \frac{6 V}{(\pi k)^{2} \omega^{n}\left(U_{k}\right)} \mathbb{1}_{U_{k}}(x) .
$$

Clearly $f \in L^{1}(X)$ with $\int_{X} f \omega^{n}=V$, hence by [23, Theorem A] there exists $v \in \mathcal{E}^{L^{1}}$ such that $\omega_{v}^{n}=f \omega^{n}$. We claim that $v \notin \mathcal{E}^{p^{\prime}}$. Indeed, if this were not true, then [23, Theorem $\left.\mathrm{C}\right]$ would give that

$$
\infty=\frac{6 V}{\pi^{2}} \sum_{k \geq 1} \frac{1}{k} \leq \int_{X}|u|^{p^{\prime}} \omega_{v}^{n}<\infty,
$$

a contradiction. Finally, as $v \in \mathcal{E}^{L^{1}} \backslash \mathcal{E}^{p^{\prime}}$, the same argument as in the previous step yields now a $d_{p, 1}^{C}$-Cauchy sequence $\left\{v_{j}\right\}_{j} \subset \mathcal{H}_{\omega}$ for which $d_{p, 1}^{C}\left(v_{j}, v\right) \rightarrow 0$, without any $d_{p^{\prime}}^{M}$-Cauchy subsequences. 
Finally, to argue (ii), we have to use jointly the $d_{p, q}^{C}$-convergence criteria (3) and the estimates of Kolodziej [25, p. 668], according to which $d_{p, q}^{C}$-convergence implies $C^{0}$-convergence of potentials. According to the $d_{p^{\prime}}^{M}$-convergence criteria (5), $C^{0}$-convergence in turn implies $d_{p^{\prime}}^{M}$-convergence, finishing the proof.

\subsection{Proof of Theorem 1.6}

The following basic consequence of the dominated convergence theorem will be used shortly:

Lemma 3.1. Suppose $f \geq 0$ such that $\int_{X} f \log (f) \omega^{n}<\infty$. Then there exists $\tilde{f}_{k} \in C^{\infty}(X)$ such that $\tilde{f}_{k}>0, \int_{X} \mid f-$ $\tilde{f}_{k} \mid \omega^{n} \rightarrow 0$ and $\int_{X} f\left(\log (f)-\log \left(\tilde{f}_{k}\right)\right) \omega^{n} \rightarrow 0$.

Proof. Let $f^{m}=\max \{\min \{f, m\}, 1 / m\}$. By the dominated convergence theorem every sequence $\left\{g_{j}\right\}_{j} \subset C^{\infty}(X)$ satisfying $m>g_{j}>1 / m$ and $\int_{X}\left|f^{m}-g_{j}\right| \omega^{n} \rightarrow 0$ contains an element $\tilde{f}_{m}:=g_{j_{m}}$ with $\int_{X} f\left(\log \left(f^{m}\right)-\log \left(\tilde{f}_{m}\right)\right) \omega^{n} \leq$ $1 / n$ and $\int_{X}\left|f^{m}-\tilde{f}_{m}\right| \omega^{n} \leq 1 / n$. By the absolute continuity of the Lebesgue integral, it follows that $\left\{\tilde{f}_{k}\right\}_{k}$ satisfies the properties of the lemma.

Proof of Theorem 1.6. First we show the equivalence between (i), (ii) and (iii). From [18, Theorem 5(i)] it follows that (iii) $\rightarrow$ (i) and (iii) $\rightarrow$ (ii).

The proof of (i) $\rightarrow$ (iii) and (ii) $\rightarrow$ (iii) are almost the same and we only carry out the latter. It follows from the compactness theorem [7, Theorem 2.17] (for a statement most suitable for our purposes see [20, Theorem 5.6]) that any subsequence of $\left\{u_{j}\right\}$ contains a subsubsequence $\left\{u_{j_{k}}\right\}$ such that $d_{1}\left(u_{j_{k}}, v\right) \rightarrow 0$ for some $v \in \mathcal{E}^{\text {Ent }}$. If we can show that $v=u$ then we are done. By [18, Theorem 5(i)] again, we have $\omega_{u_{j}}^{n} \rightarrow \omega_{v}^{n}$ weakly, hence by the assumption we get $\omega_{u}^{n}=\omega_{v}^{n}$. As $u, v \in \mathcal{E}^{1}$, by uniqueness [23, Theorem B] (see [21] for a more general result), we conclude that $v=u$.

The direction (iv) $\rightarrow$ (ii) is trivial and the main step is to argue that (ii) $\rightarrow$ (iv). Let $f_{j}=\omega_{u_{j}}^{n} / \omega^{n}$ and $f=\omega_{u}^{n} / \omega^{n}$. By the $d_{p, 1}^{C}$-convergence criteria (3), we have to show that $\int_{X}\left|f-f_{j}\right| \omega^{n} \rightarrow 0$. Let $\tilde{f}_{j} \in C^{\infty}(X)$ be the sequence from the previous lemma. For fixed $k$ we have

$$
\lim _{j} \int_{X}\left|f-f_{j}\right| \omega^{n} \leq \limsup \int_{X}\left|\tilde{f}_{k}-f_{j}\right| \omega^{n}+\int_{X}\left|f-\tilde{f}_{k}\right| \omega^{n},
$$

hence we only need to check that the first term on the right hand side goes to zero as $k \rightarrow \infty$. Using the classical Küllback-Pinsker inequality (see [7, Proposition 2.10(ii)] for statement tailored to our setting) we have the following sequence of estimates:

$$
\begin{aligned}
\limsup _{j}\left(\int_{X}\left|f_{j}-\tilde{f}_{k}\right| \omega^{n}\right)^{2} & \leq \limsup _{j} \int f_{j} \log \left(\frac{f_{j}}{\tilde{f}_{k}}\right) \omega^{n} \\
& \leq \limsup _{j} \int f_{j} \log f_{j} \omega^{n}-\liminf _{j} \int f_{j} \log \tilde{f}_{k} \omega^{n} \\
& =\int f \log f \omega^{n}-\int f \log \tilde{f}_{k} \omega^{n},
\end{aligned}
$$

where to get the last line we have used that $\operatorname{Ent}\left(\omega_{u_{j}}, \omega\right) \rightarrow \operatorname{Ent}\left(\omega_{u}, \omega\right)$ and that $\omega_{u_{j}}^{n}=f_{j} \omega^{n}$ converges weakly to $\omega_{u}^{n}$. By the previous lemma, the expression in (8) tends to zero as $k \rightarrow \infty$, hence we are done.

It is perhaps worth noting that (iv) implies (ii) in Theorem 1.6 without the assumption on the convergence of entropy, as we elaborate now. Suppose $u_{j}, u \in \mathcal{E}^{L^{1}}$ with $d_{p, 1}^{M}\left(u_{j}, u\right) \rightarrow 0$. As $\left\{u_{j}\right\}_{j}$ is $L^{1}\left(X, \omega^{n}\right)$-compact (since $\left.\int_{X} u_{j} \omega^{n}=0\right)$, we have to argue that any $L^{1}(X, \omega)$-convergent subsequence of $\left\{u_{j}\right\} L^{1}$-converges to $u$. Suppose $u_{j_{k}} \rightarrow_{L^{1}} v \in \operatorname{PSH}(X, \omega)$. By [5, Proposition 2.10(i)] it follows that $v \in \mathcal{E}^{1}$, in particular $\omega_{v}^{n}$ has full mass $(u \in \mathcal{E})$. As a consequence of [5, Corollary 2.21] we now obtain that $\omega_{v}^{n} \geq \omega_{u}^{n}$. As both of these last measures have the same total volume we have in fact $\omega_{v}^{n}=\omega_{u}^{n}$, hence $v=u$ as desired (here we used again [23, Theorem B]). 
For $q>1, d_{p, q^{-}}^{C}$-convergence implies $C^{0}$-convergence (hence also $L^{1}\left(X, \omega^{n}\right)$-convergence of potentials) as was noted in the proof of Theorem 1.5, and we summarize our findings in the next remark, obtaining a partial analog of $\left[18\right.$, Theorem 5(i)] for the $d_{p, q}^{C}$ metric in the process:

Remark 3.2. Suppose $1 \leq q \leq p<\infty$ and $u_{j}, u \in \mathcal{E}^{L^{q}}$. Then $d_{p, q}^{C}\left(u_{j}, u\right) \rightarrow 0$ implies that $u_{j} \rightarrow_{L^{1}\left(X, \omega^{n}\right)} u$.

\section{Conflict of interest statement}

The author certifies that they have no affiliations with or involvement in any organization or entity with any financial interest, or non-financial interest in the subject matter or materials discussed in this manuscript.

\section{Acknowledgement}

This research was supported by United States - Israel Binational Science Foundation grant 2012236.

\section{References}

[1] R. Berman, S. Boucksom, M. Jonsson, A variational proof of a uniform version of the Yau-Tian-Donaldson conjecture for Fano manifolds, arXiv: 1509.04561.

[2] R. Berman, T. Darvas, L. Chinh, Convexity of the extended K-energy and convergence of the weak Calabi flow, preprint, August 2015.

[3] Z. Blocki, Uniqueness and stability for the Monge-Ampère equation on compact Kähler manifolds, Indiana Univ. Math. J. 52 (2003) 1697-1702.

[4] Z. Blocki, On uniqueness of the complex Monge-Ampère equation on compact Kähler manifolds, Institut Mittag-Leffler, Report No. 1, 2007/2008

[5] S. Boucksom, P. Eyssidieux, V. Guedj, A. Zeriahi, Monge-Ampère equations in big cohomology classes, Acta Math. 205 (2010) 199-262.

[6] Sébastien Boucksom, Philippe Eyssidieux, Vincent Guedj (Eds.), An Introduction to the Kähler-Ricci Flow, Lecture Notes in Mathematics, vol. 2086, Springer, Cham, 2013

[7] S. Boucksom, R. Berman, P. Eyssidieux, V. Guedj, A. Zeriahi, Kähler-Einstein metrics and the Kähler-Ricci flow on log Fano varieties, arXiv: 1111.7158.

[8] M. Bridson, A. Haefliger, Metric Spaces of Non-Positive Curvature, Grundlehren der Mathematischen Wissenschaften (Fundamental Principles of Mathematical Sciences), vol. 319, Springer-Verlag, Berlin, 1999.

[9] E. Calabi, The variation of Kähler metrics. I. The structure of the space; II. A minimum problem, Bull. Am. Math. Soc. 60 (1954) 167-168.

[10] E. Calabi, Extremal Kähler metrics, in: Seminar on Differential Geometry, in: Ann. Math. Stud., vol. 102, Princeton Univ. Press, Princeton, NJ, 1982, pp. 259-290.

[11] E. Calabi, X.X. Chen, The space of Kähler metrics. II, J. Differ. Geom. 61 (2) (2002) 173-193.

[12] S. Calamai, The Calabi metric for the space of Kähler metrics, Math. Ann. 353 (2) (2012) 373-402.

[13] S. Calamai, K. Zheng, The Dirichlet and the weighted metrics for the space of Kähler metrics, Math. Ann. 363 (3) (2015) $817-856$.

[14] B. Clarke, Y.A. Rubinstein, Ricci flow and the metric completion of the space of Kähler metrics, Am. J. Math. 135 (6) (2013) $1477-1505$.

[15] B. Clarke, Y.A. Rubinstein, Conformal deformations of the Ebin metric and a generalized Calabi metric on the space of Riemannian metrics, Ann. Inst. Henri Poincaré, Anal. Non Linéaire 30 (2) (2013) 251-274.

[16] T. Collins, G. Székelyhidi, The twisted Kähler-Ricci flow, arXiv:1207.5441.

[17] T. Darvas, Envelopes and geodesics in spaces of Kähler potentials, arXiv:1401.7318.

[18] T. Darvas, The Mabuchi geometry of finite energy classes, Adv. Math. 285 (2015) 182-219.

[19] T. Darvas, W. He, Geodesic rays and Kähler-Ricci trajectories on Fano manifolds, arXiv:1411.0774.

[20] T. Darvas, Y. Rubinstein, Tian's properness conjectures and Finsler geometry of the space of Kähler metrics, arXiv:1506.07129.

[21] S. Dinew, Uniqueness in $\mathcal{E}(X, \omega)$, J. Funct. Anal. 256 (7) (2009) 2113-2122.

[22] S.K. Donaldson, Symmetric spaces, Kähler geometry and Hamiltonian dynamics, in: Amer. Math. Soc. Transl. Ser. 2, vol. 196, Amer. Math. Soc., Providence, RI, 1999, pp. 13-33.

[23] V. Guedj, A. Zeriahi, The weighted Monge-Ampère energy of quasiplurisubharmonic functions, J. Funct. Anal. 250 (2) (2007) $442-482$.

[24] R.S. Hamilton, Three-manifolds with positive Ricci curvature, J. Differ. Geom. 17 (1982) 255-306.

[25] S. Kolodziej, The Monge-Ampère equation on compact Kähler manifolds, Indiana Univ. Math. J. 52 (3) (2003) $667-686$.

[26] T. Mabuchi, Some symplectic geometry on compact Kähler manifolds I, Osaka J. Math. 24 (1987) 227-252.

[27] D. McFeron, The Mabuchi metric and the Kähler-Ricci flow, Proc. Am. Math. Soc. 142 (3) (2014) 1005-1012.

[28] D.H. Phong, J. Song, J. Sturm, B. Weinkove, The Kähler-Ricci flow and the $\bar{\partial}$-operator on vector fields, J. Differ. Geom. 81 (2009) $631-647$.

[29] I.K. Rana, An Introduction to Measure and Integration, second edition, Graduate Studies in Mathematics, vol. 45, American Mathematical Society, Providence, RI, 2002.

[30] Y.A. Rubinstein, On the construction of Nadel multiplier ideal sheaves and the limiting behavior of the Ricci flow, Trans. Am. Math. Soc. 361 (2009) 5839-5850.

[31] S. Semmes, Complex Monge-Ampère and symplectic manifolds, Am. J. Math. 114 (1992) 495-550. 
[32] J. Streets, Long time existence of minimizing movement solutions of Calabi flow, Adv. Math. 259 (2014) 688-729.

[33] J. Streets, The consistency and convergence of K-energy minimizing movements, arXiv:1301.3948.

[34] G. Tian, X.H. Zhu, Convergence of Kähler-Ricci flow, J. Am. Math. Soc. 20 (2007) 675-699.

[35] S.T. Yau, On the Ricci curvature of a compact Kähler manifold and the complex Monge-Ampère equation, I, Commun. Pure Appl. Math. 31 (1978) 339-411. 